\title{
On the Activity of the Corticostriatal Networks during Spike-and-Wave Discharges in a Genetic Model of Absence Epilepsy
}

\author{
Seán J. Slaght, ${ }^{1,2}$ Tamar Paz, ${ }^{1}$ Mario Chavez, ${ }^{3}$ Jean-Michel Deniau, ${ }^{1}$ Séverine Mahon, ${ }^{1}$ and Stéphane Charpier ${ }^{1}$ \\ ${ }^{1}$ Institut National de la Santé et de la Recherche Médicale U114, Chaire de Neuropharmacologie, Collège de France, 75231 Paris Cedex 05, France, ${ }^{2}$ Cardiff \\ School of Biosciences, Cardiff University, Cardiff CF10 3US, United Kingdom, and '3aboratoire de Neurosciences Cognitives et d’Imagerie Cérébrale, \\ Hôpital de la Pitié Salpétrière, Centre National de la Recherche Scientifique, Unité Propre de Recherche 640, 75651 Paris Cedex 13, France
}

\begin{abstract}
Absence seizures are characterized by impairment of consciousness associated with widespread bilaterally synchronous spike-and-wave discharges (SWDs) in the electroencephalogram (EEG), which reflect highly synchronized oscillations in thalamocortical networks. Although recent pharmacological studies suggest that the basal ganglia could provide a remote control system for absence seizures, the mechanisms of propagation of epileptic discharges in these subcortical nuclei remain unknown. In the present study, we provide the first description of the electrical events in the corticostriatal pathway during spontaneous SWDs in the genetic absence epilepsy rats from Strasbourg (GAERS), a genetic model of absence epilepsy. In corticostriatal neurons, the SWDs were associated with suprathreshold rhythmic depolarizations in-phase with local EEG spikes. Consistent with this synchronized firing in their excitatory cortical afferents, striatal output neurons (SONs) exhibited, during SWDs, large-amplitude rhythmic synaptic depolarizations. However, SONs did not discharge during SWDs. Instead, the rhythmic synaptic excitation of SONs was shunted by a $\mathrm{Cl}^{-}$-dependent increase in membrane conductance that was temporally correlated with bursts of action potentials in striatal GABAergic interneurons. The reduced SON excitability accompanying absence seizures may participate in the control of SWDs by affecting the flow of cortical information within the basal ganglia circuits.
\end{abstract}

Key words: basal ganglia; corticostriatal neurons; GABAergic interneurons; GAERS; striatum; shunting inhibition

\section{Introduction}

Absence epilepsy is an idiopathic, nonconvulsive, generalized epilepsy of multifactorial origin. Absence seizures consist of a sudden impairment of consciousness concomitant with bilateral synchronized spike-and-wave discharges (SWDs) in the electroencephalogram (EEG) over wide cortical areas (Panayiotopoulos, 1997). Electrophysiological recordings in patients (Williams, 1953 ) and in various animal models of absence epilepsy (for review, see Danober et al., 1998; Crunelli and Leresche, 2002) revealed that SWDs reflect highly synchronized oscillations in thalamocortical networks.

Recent pharmacological studies performed in genetic absence epilepsy rats from Strasbourg (GAERS), a well established genetic

Received April 17, 2004; revised June 7, 2004; accepted June 9, 2004.

This work was supported by the Ministère Français de la Recherche (Action Concertée d'Initiative du Développement et Physiologie Intégrative 2000) and the Département of Sciences et Technologies de l'Information et de la Communication de l'Institut National de la Santé et de la Recherche Médicale (2003). We thank Dr. R. Miles and Prof. V. Crunelli for thoughtful discussion and critical reading of this manuscript.

S.J.S. was a Wellcome Prize Student.

Correspondence should be addressed to $\mathrm{S}$. Charpier, Institut National de la Santé et de la Recherche Médicale U114, Chaire de Neuropharmacologie, Collège de France, 11 Place Marcelin Berthelot, 75231 Paris Cedex 05, France. E-mail: stephane.charpier@college-de-france.fr.

S. Mahon's present address: Wolfson Institute for Biomedical Research and Department of Physiology, University College London, Gower Street, London WC1E 6BT, UK.

DOI:10.1523/JNEUROSCI.1449-04.2004

Copyright $\odot 2004$ Society for Neuroscience $\quad$ 0270-6474/04/246816-10\$15.00/0 model of absence epilepsy (Marescaux et al., 1992; Danober et al., 1998), indicate that the basal ganglia circuits could act as a remote control system for absence seizures via a modulation of activity in the striatonigral pathway (Depaulis et al., 1988, 1989; Danober et al., 1998; Deransart et al., 1998, 2000, 2001; Deransart and Depaulis, 2002). Specifically, the frequency of absence seizures is significantly decreased by injection of $\mathrm{GABA}_{\mathrm{A}}$ receptor agonists into the substantia nigra pars reticulata $(\mathrm{SNr})$ or by the activation of GABAergic striatonigral neurons after intrastriatal injection of NMDA or dopaminergic $D_{1}$ receptor agonists (Depaulis et al., 1988; Deransart et al., 1998). Conversely, the pharmacological blockade of $\mathrm{GABA}_{\mathrm{A}}$ receptors in the $\mathrm{SNr}$ or the blockade of striatal $\mathrm{D}_{1}$ receptors aggravates absence seizures (Deransart et al., $2000,2001)$. These findings suggest that an increase or a decrease in the activity of GABAergic striatal output neurons (SONs) would attenuate or amplify, respectively, the occurrence of abnormal thalamocortical oscillations.

Synaptic inputs to SONs include glutamatergic excitatory inputs arising from a large number of convergent corticostriatal (CS) neurons (Wilson, 1995; Kincaid et al., 1998; Zheng and Wilson, 2002) and inhibitory connections originating from both collateral interactions between SONs (Czubayko and Plenz, 2002; Tunstall et al., 2002) and GABAergic striatal interneurons (Plenz and Kitai, 1998; Koós and Tepper, 1999), which also receive powerful excitatory cortical inputs (Kita, 1993; Ramanathan et al., 
2002). Given the functional impact of the corticostriatal projections on the excitability of SONs, the cortical paroxysms associated with absence seizures could produce significant changes in the firing pattern of SONs, which could lead to positive or negative modulation of SWDs.

Therefore, an important step in elucidating the role of the basal ganglia in the control of absence seizures is to determine how these epileptic activities propagate from the cortex to the striatum. However, the impact of cortical SWDs on the activity of SONs remains unknown, and electrical changes occurring in the principal neuronal elements of the corticostriatal pathway during absence seizures have not been characterized. In the present study, we investigated this issue in vivo in GAERS by examining the intracellular and/or extracellular electrical activity of corticostriatal neurons, SONs, and GABAergic striatal interneurons, simultaneously with the spontaneous SWDs occurring in the EEG of the related cortical region.

\section{Materials and Methods}

All experiments were performed in accordance with local Ethical Committee and European Union guidelines (directive 86/609/EEC), and every precaution was taken to minimize stress and the number of animals used in each series of experiments.

Animal preparation. Experiments were performed in vivo on 15 adult $(4-5$ month old) male $(n=9)$ and female $(n=6)$ rats from the GAERS strain. Animals were initially anesthetized with sodium pentobarbital (40 $\mathrm{mg} / \mathrm{kg}$, i.p.; Sanofi, Libourne, France) and ketamine $(100 \mathrm{mg} / \mathrm{kg}$, i.m.; Imalgène, Rhone Mérieux, France). A cannula was inserted into the trachea, and the animal was placed in a stereotaxic frame. Wounds and pressure points were repeatedly (every $2 \mathrm{hr}$ ) infiltrated with lignocaine $(2 \%)$. Once the surgical procedures had been completed (see below), ear bars were removed, and the head was held via a metallic rod cemented to the skull. Rats were subsequently maintained in a narcotized and sedated state by injections of fentanyl ( $3 \mu \mathrm{g} / \mathrm{kg}$, i.p.; Janssen-Cilag, Issy-LesMoulineaux, France) repeated every 20-30 min (Simons and Carvell, 1989; Pinault et al., 1998; Charpier et al., 1999; Slaght et al., 2002a; Bruno et al., 2003). To obtain long-lasting stable intracellular recordings, rats were immobilized with gallamine triethiodide $(40 \mathrm{mg}$, i.m., every $2 \mathrm{hr}$; Specia, Paris, France) and artificially ventilated. The degree of anesthesia was assessed by continuously monitoring the EEG and heart rate, and additional doses of fentanyl were administered at the slightest change toward an awaked pattern (i.e., an increase in the frequency and reduction in the amplitude of the EEG waves and/or an increase in the heart rate). Body temperature was maintained $\left(36.5-37.5^{\circ} \mathrm{C}\right)$ with a homeothermic blanket. At the end of the experiments, animals received an overdose of sodium pentobarbital $(200 \mathrm{mg} / \mathrm{kg}$, i.p.).

Electrophysiological recordings. EEG recordings were obtained with a low-impedance $(\approx 60 \mathrm{~K} \Omega)$ silver electrode placed on the dura above the orofacial motor cortex (12 mm anterior to the interaural line; $3.5-4 \mathrm{~mm}$ lateral to the midline) (Neafsey et al., 1986), and the reference electrode was placed in the muscle to the opposite side of the head.

Intracellular recordings were performed using glass micropipettes filled with $2 \mathrm{M}$ potassium acetate $(50-70 \mathrm{M} \Omega)$ or $3 \mathrm{M} \mathrm{KCl}(30-40 \mathrm{M} \Omega$ ). Measurements of apparent membrane input resistance and time constant were based on the linear electrical cable theory applied to an idealized isopotential neuron (Rall, 1969). Apparent input resistance was assessed by measurement of the mean $(n \geq 10)$ membrane potential change at the end of hyperpolarizing current pulses of low intensity $(-0.4 \mathrm{nA}, 200 \mathrm{msec}$ duration, every $1.55 \mathrm{sec})$ applied through the recording electrode, and the membrane time constant was the time taken for the membrane potential to reach $63 \%$ of its final value. In the case of membrane rectification, the input resistance was assessed from the linear portion of the current-voltage $(I-V)$ relationship (see Fig. 3). The values of membrane potential were corrected according to the potential recorded extracellularly immediately after termination of the intracellular recording.
For single-cell extracellular recordings and juxtacellular labeling (see below), glass electrodes were filled with $0.5 \mathrm{M} \mathrm{NaCl}$ and $1.5 \%$ neurobiotin (15-20 M $\Omega$; Vector Laboratories, Burlingame, CA). Cortical cells, located within the orofacial motor cortex, were recorded within $300 \mu \mathrm{m}$ of the EEG electrode at the following coordinates: $12 \mathrm{~mm}$ anterior to the interaural line, $3.6-3.8 \mathrm{~mm}$ lateral to the midline, and $1.2-2 \mathrm{~mm}$ under the cortical surface. The cortical cells were electrophysiologically identified as CS neurons by their antidromic activation after electrical stimulation within the contralateral striatum $(8.7-9 \mathrm{~mm}$ anterior to the interaural line; $3.5-4 \mathrm{~mm}$ lateral to the midline; $5.2-5.4 \mathrm{~mm}$ ventral to the brain surface). Striatal stimuli used to test antidromic activation (200 $\mu$ sec duration; 5-20 V) were applied with a bipolar concentric electrode (NE-100; Rhodes Medical Instruments, Woodland Hills, CA). The criteria used for identification of antidromic action potentials were as follows: (1) the constant latency of the antidromic response despite imposed changes of membrane potential, (2) collision of the antidromic spikes with spontaneously occurring orthodromic action potentials, and (3) the all-or-none property of the evoked spikes when the stimulation was just below threshold for antidromic activation (see Fig. $1 A$ ).

Striatal intracellular (SONs) and single-cell extracellular (SONs and GABAergic interneurons) recordings were obtained from the striatal projection field of the orofacial motor cortex (Deniau et al., 1996). The corresponding stereotaxic coordinates were as follows: $9 \mathrm{~mm}$ anterior to the interaural line, $3.5-4 \mathrm{~mm}$ lateral to the midline, and $3-5.6 \mathrm{~mm}$ ventral to the brain surface.

In all experiments, the intracellular or single-cell extracellular recordings were simultaneously performed with the corresponding ipsilateral cortical EEG.

Morphological identification. Extracellularly recorded neurons were labeled by juxtacellular injection of neurobiotin (Pinault, 1996; Mailly et al., 2003). Briefly, positive current pulses (1-8 nA, $200 \mathrm{msec}$ ) were applied at a frequency of $2.5 \mathrm{~Hz}$ through the bridge circuit of the amplifier. The current was slowly increased while the electrode was advanced toward the neuron in $1 \mu \mathrm{m}$ steps (LSS-1000 Inchworm Motor Positioning System; Burleigh Instruments, Fishers, NY) until the cell discharge was driven by the injected current. Current pulses were applied for a 10-15 min period to obtain a reliable labeling of neuronal processes (see Figs. $2 A 1,8 A 1$ ). The histochemical methods used to reveal the morphology of neurobiotin-filled neurons were described in detail previously (Slaght et al., 2002a).

Data acquisition and analysis. Intracellular and single-cell extracellular recordings were obtained under current-clamp conditions using the active bridge mode of an Axoclamp-2B amplifier (Axon Instruments, Union City, CA). Data were stored on-line on a DRA 800 digital tape recorder (Biologic, Claix, France) and then digitized with a sampling rate of $20 \mathrm{kHz}$ (intracellular signals), $10 \mathrm{kHz}$ (extracellular signals), or $300 \mathrm{~Hz}$ (EEG) for off-line analysis. To perform spectral analysis of EEG potentials, fast Fourier transforms were applied using Spike 2 (CED Software; Cambridge Electronic Design, Cambridge, UK). The amplitude of action potentials was calculated as the potential difference between their voltage threshold, measured as the membrane potential at which the $d V / d t$ exceeded $10 \mathrm{~V} / \mathrm{sec}^{-1}$ (Mahon et al., 2003), and the peak of the spike waveform. Numerical values are given as means \pm SEM unless stated otherwise. Statistical significance was assessed by performing appropriate statistical tests, Student's $t$ test, or a one-way ANOVA. In some measurements, the normality of distributions was tested using the KolmogorovSmirnov test, and a Gaussian-Laplace fit was performed. Statistical analysis and curve fitting were performed with Origin 6.0 (Microcal Software, Northampton, MA).

To quantify the degree of statistical association and the time delay between EEG waves and intracellular activity of SONs, the nonlinear correlation coefficient $h^{2}$ was calculated between these signals as a function of a time shift $(\tau)$ (Lopes da Silva et al., 1989). Although classical methods such as cross-correlation or coherence yield reliable results only for linearly related signals, this nonlinear index can reveal signal interdependencies under more general conditions (Meeren et al., 2002). 
To quantify the dependence of a signal $Y$ on a signal $X$, an estimator of the nonlinear correlation index $\left(h^{2}\right)$ was computed as follows:

$h^{2}(x \rightarrow y)$

$$
=\frac{\sum_{i=1}^{N}\left(y_{i}-\langle y\rangle\right)^{2}-\sum_{i=1}^{N}\left(y_{i}-g\left(x_{i}\right)\right)^{2}}{\sum_{i=1}^{N}\left(y_{i}-\langle y\rangle\right)^{2}}
$$

where $g\left(x_{i}\right)$ denotes a piecewise linear regression curve, used to predict the signal $Y$ given the values of $X$, and $\langle y\rangle$ denotes the average of the time series $y_{i}$ over $N$ samples. Statistically, this nonlinear correlation index quantifies the reduction of variance that can be obtained by predicting the $Y$ values on the basis of the regression curve. Theoretical and practical aspects of this method were described in detail previously (Lopes da Silva et al., 1989).

The nonlinear index ranges between 0 , when both signals are independent, and 1, for a perfect dependence. This procedure, in contrast to "classical" linear correlation functions, may yield an asymmetric function, [i.e., $h^{2}(x \rightarrow y) \neq$ $h^{2}(y \rightarrow x)$ ], which may provide insights into possible driving-response relationship (Lopes da Silva et al., 1989).

In the present study, the degree of association between EEG and intracellular signals and the corresponding time delays during seizures were obtained by computing $h^{2}$ as a function of a time shift $(\tau)$ between the signals $y$ and $x$. The shift for which the maximum of $h^{2}(\tau)$ was reached provided an estimate of the time delay between the activities (Meeren et al., 2002). Because epileptic activities are not stationary in time, the correlation indices were computed for sliding short epochs of $2 \mathrm{sec}$ with $98 \%(\approx 1.96 \mathrm{sec})$ of temporal overlap.

\section{Results \\ SWD properties}

The intra-SWD frequency, revealed by spectral analysis of the EEG signal, ranged from 7 to $9.7 \mathrm{~Hz}(7.9 \pm 0.1 \mathrm{~Hz} ; n=517$ SWDs from 13 rats) (Fig. 1C). SWDs had a duration of $7 \pm 1.3 \mathrm{sec}$ (from 0.6 to $23.1 \mathrm{sec})$ and recurred once every $9-260 \mathrm{sec}(55.4 \pm 26 \mathrm{sec})$. These temporal properties of SWDs, as well as the shape of individual spike-wave complexes, are similar to those described previously under analogous experimental conditions (Pinault et al., 1998; Charpier et al., 1999; Slaght et al., 2002a,b; Pinault, 2003) and in freely moving GAERS (Marescaux et al., 1992).

\section{Intracellular activity of CS neurons}

We recorded from CS neurons $(n=4)$ located in the orofacial motor cortex. The depth of intracellular recordings, between 1200 and $2000 \mu \mathrm{m}$ from the cortical surface, suggested that CS neurons were located in the deep part of layer III and in layer V. This laminar localization is consistent with previous findings obtained from intracellular labeling of crossed CS neurons (Cowan and Wilson, 1994). CS neurons were electrophysiologically identified by antidromic activation after electrical stimulation of the contralateral striatum (Fig. $1 A$ ). Antidromic latencies ranged between 3.2 and $8.6 \mathrm{msec}(6.4 \pm 1.15 \mathrm{msec} ; n=4)$, and the passive membrane properties of CS neurons, measured during interictal periods, included a membrane potential of $-61.3 \pm 3.1 \mathrm{mV}(n=$ $4)$ and an apparent input resistance of $23 \pm 3.4 \mathrm{M} \Omega(n=4)$. Action potentials of CS neurons were overshooting, and the firing pattern evoked by intracellular injection of positive square current pulses (Fig. 1 $A$, inset) was characteristic of "regularspiking" neocortical neurons (Connors and Gutnick, 1990). These electrophysiological properties of CS neurons agree with those measured previously in crossed CS neurons from nonepileptic rats (Cowan and Wilson, 1994; Mahon et al., 2001).

During SWDs, CS neurons exhibited sudden rhythmic membrane depolarizations (Fig. $1 B$ ), which were temporally correlated with the spike-wave complexes (Fig. $1 E$, inset). These repetitive membrane depolarizations, which could trigger action potentials, were superimposed on a tonic membrane hyperpolarization (5-15 $\mathrm{mV}$ in amplitude) that lasted for the entire SWD (Fig. $1 B$ ). The mean spontaneous firing frequency of CS neurons (Fig. 1D) was increased during the SWDs compared with the interictal periods (interictal, $3.3 \pm 1.3 \mathrm{~Hz}$ vs SWDs, $4.4 \pm 1 \mathrm{~Hz}$; $n=4$ cells; $p<0.05)$. To assess the temporal relationship between the firing of CS neurons and the cortical paroxysms during absence seizures, we measured the timing of individual action potentials in CS neurons relative to the peak negativity of the corresponding spike component in the EEG (Fig. 1 E, inset). Action potentials in CS neurons typically preceded the EEG spike component (Fig. $1 E$ ) with a mean latency of $-19 \pm 1.9 \mathrm{msec}$ ( $n=200$ action potentials from four cells). 

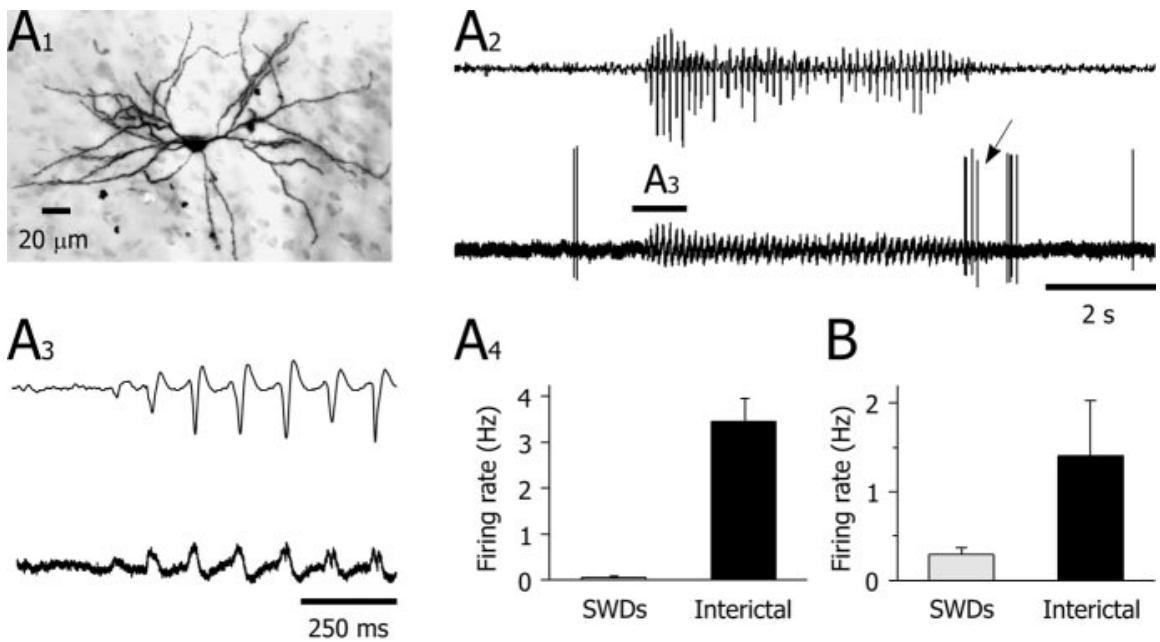

$\mathrm{A}_{4}$

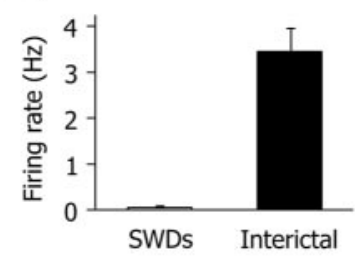

B

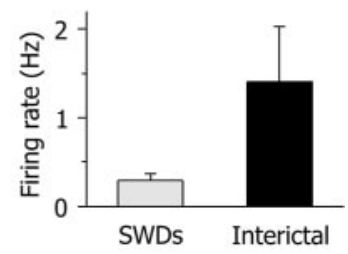

Figure 2. Extracellularly recorded activity of SON during SWDs. A1, Synthetic projection micrograph (from a 400- $\mu \mathrm{m}$-thick whole mount) of a striatal output neuron labeled by juxtacellular injection of neurobiotin. This cell exhibited the characteristic morphological features of striatal output neurons (for a detailed description, see Results). A2, The occasional spontaneous firing of the striatal cell (bottom trace) was interrupted during the SWD (top trace). An oscillatory field potential was maintained throughout the crisis. Note the rebound of firing at the end of the SWD (oblique arrow). A3, The EEG record (top trace) and the corresponding striatal field potential (bottom trace) during the start of the SWD (as shown in A2). A4, The mean spike firing frequency calculated from the interictal periods ( $n=11)$ was dramatically decreased during the SWDs $(n=10)(p<0.001)$. B, Pooled data from 10 cells showing the significant $(p<0.05)$ diminution of the mean spontaneous firing rate of striatal output neurons during absence seizures. Results depicted in $A 1-A 4$ are from the same cell.

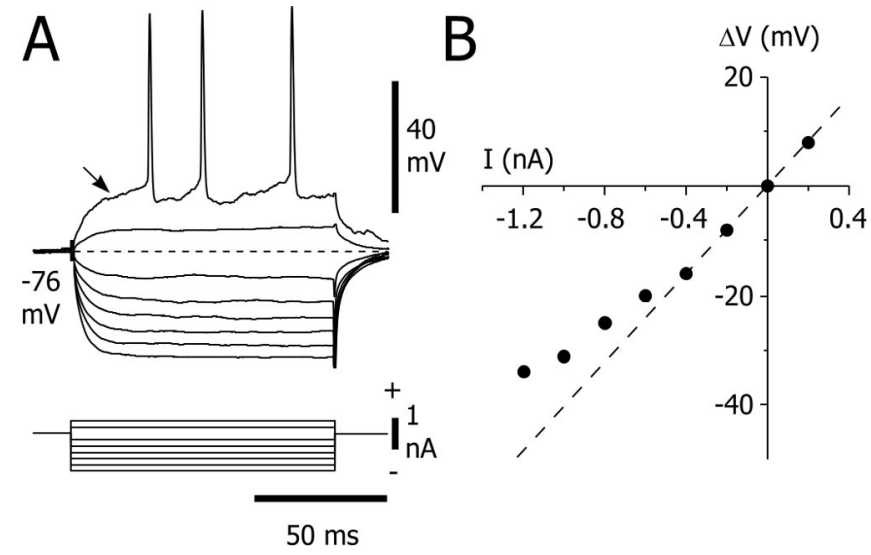

Figure 3. Electrical membrane properties of GAERS striatal output neurons. A, Voltage responses of a striatal output neuron (top traces) to a series of hyperpolarizing and depolarizing square current pulses (bottom traces). The traces represent the average of 15 successive trials, except the suprathreshold response evoked by a single positive current pulse. Note the slow ramp-like depolarization (arrow) that preceded the action potential discharge. The value of the resting membrane potential (dashed line) is indicated. $B$, The plot of voltage changes $(\Delta V)$ as a function of the injected current $(I)$ was constructed from the responses shown in $A$. The apparent input resistance of this cell ( $40 \mathrm{M} \Omega$ ) was calculated from the linear segment (dashed line) of the $I-V$ curve. Note the rectification of the membrane potential in response to hyperpolarizing currents less than $-0.4 \mathrm{nA}$.

\section{Extracellular recording of SONs}

Changes in the electrical activity of SONs during SWDs were first examined by extracellular recordings ( $n=10$ cells). These cells, which form the majority of striatal neurons (Chang et al., 1982), were morphologically identified by juxtacellular injection of neurobiotin (see Materials and Methods). Labeled cells (Fig. 2A1) were located in the striatal projection field of the orofacial motor cortex and displayed the distinctive morphological features of SONs, i.e., those of the type I medium spiny cells (Chang et al., 1982; Kawaguchi, 1993). Briefly, they possessed somata of diam- eter between 10 and $20 \mu \mathrm{m}$, and their dendrites were densely covered with spines apart from their most proximal regions.

The spontaneous firing rate of SONs was dramatically reduced during SWDs. Between cortical paroxysms, SONs discharged action potentials intermittently at a mean frequency of $1.41 \pm 1.97 \mathrm{~Hz}$ (from 0.022 to $4.9 \mathrm{~Hz} ; n=10$ cells) (Fig. $2 A 2, A 4, B)$. This firing rate was decreased by $79.4 \%$ during SWDs, reaching a mean value of $0.29 \pm 0.22 \mathrm{~Hz}$ (from 0.02 to 0.68 $\mathrm{Hz} ; n=10$ cells) (Fig. $2 A 2, A 4, B$ ), which corresponded to a probability of firing in association with individual spike-wave complexes of $0.04 \pm 0.003$ (from 0.004 to $0.1 ; n=10$ cells). In most neurons $(n=6$ of 10), the strong reduction in firing during the ictal epochs was followed by a rebound of excitation manifested by either single or short bursts of two to five action potentials (Fig. 2A2, oblique arrow). As illustrated in Figure 2, A2 and A3, SWDs were accompanied by small-amplitude rhythmic striatal field potentials. This finding strongly suggested that the ictal cortical oscillations, which were associated with rhythmic firing in CS neurons, generated synchronized synaptic oscillations within the striatum. This hypothesis was also supported by the temporal relationships and the degree of association between SWDs and intracellular oscillations in SONs (see below).

Our extracellular recordings provide little information on the behavior of inactive neurons. However, we never recorded extracellular units that discharged action potentials exclusively during SWDs, providing evidence against the recruitment of a striatal neuron population during paroxysmal activity. This hypothesis was confirmed by the intracellular recordings of SONs (see below).

\section{Intracellular activity of SONs}

Because action potential firing in SONs is naturally and consistently initiated by excitatory synaptic potentials generated by synchronized corticostriatal inputs (Wilson, 1995; Wilson and Kawaguchi, 1996; Stern et al., 1997; Mahon et al., 2001), this absence of firing during SWDs was unexpected. Therefore, we performed intracellular records $(n=10)$ to explore cellular and synaptic mechanisms controlling SON activity during absence seizures. We first examined the membrane $I-V$ relationship of SONs to test for changes in their intrinsic excitability, which could account for their apparent hypoexcitability during SWDs. The membrane $I-V$ relationship was obtained, during interictal periods, by measuring membrane potential changes in response to a series of intracellular square current pulses (Fig. $3 A$ ). The apparent input resistance, measured from the linear portion of the $I-V$ plot (Fig. $3 B$, dashed line) was $33 \pm 2 \mathrm{M} \Omega$ (from 25 to 40 $\mathrm{M} \Omega ; n=10$ cells), and a marked inward rectification was consistently observed in response to current pulses of increasingly negative intensity (Fig. 3B). Membrane time constants ranged between 3.1 and $7.7 \mathrm{msec}(4.9 \pm 0.5 \mathrm{msec} ; n=10$ cells $)$. Suprathreshold positive current pulses evoked a slow ramp-like membrane depolarization (Fig. $3 A$, arrow) that led to a delay in the first action potential discharge. Action potentials of SONs had 
an amplitude and duration of $55.4 \pm 1.9$ $\mathrm{mV}$ and $1.6 \pm 0.1 \mathrm{msec}(n=10$ cells $)$, respectively, and a voltage threshold of $-50 \pm 0.2 \mathrm{mV}(n=10$ cells $)$. Membrane potential of SONs, measured between SWDs, was $-76.8 \pm 1.4 \mathrm{mV}(n=10$ cells $)$. These membrane properties are similar to those described previously in vivo from nonepileptic rats (Wilson, 1995; Charpier et al., 1999; Mahon et al., 2001, 2003). This finding suggests that changed neuronal membrane properties did not underlie the inability of GAERS striatal output neurons to fire action potentials during SWDs. Furthermore, no striking differences were evident in synaptic events that impinged on SONs between paroxysms. Spontaneous events were composed of highfrequency, small-amplitude, depolarizing synaptic potentials (Figs. $4 A, 5 A, 6 A$ ) resembling those recorded in vivo in normal rats under fentanyl (Mahon et al., 2001). This intracellular activity of SONs noticeably differs from the two-state behavior observed under ketamine-xylazine anesthesia, i.e., characteristic shifts of membrane potential between a hyperpolarized downstate and depolarized upstate (Wilson, 1995; Wilson and Kawaguchi, 1996; Stern et al., 1997; Mahon et al., 2001), which are associated with rhythmic largeamplitude EEG waves (Mahon et al., 2001).

Analysis of the intracellular firing pattern of SONs during SWDs confirmed and extended the observations made during extracellular recordings. Of the 10 intracellularly recorded cells, seven were silent throughout the recording session, including the epochs of cortical paroxysms. In the three remaining neurons, which exhibited a low interictal mean firing rate (from 0.04 to $1 \mathrm{~Hz}$ ), two were silenced by the cortical SWDs and one displayed a probability of firing, as low as 0.01 , in association with the spike-wave complexes. Membrane potential and apparent input resistance of silent and weakly active SONs did not differ significantly ( $p>0.1$ for each parameter), indicating that the slight difference of firing among SONs was not attributable to distinct intrinsic membrane properties. In addition, the fact that extracellularly recorded SONs can be silenced by the SWDs (Fig. 2A2) demonstrates that cell impalement was not responsible for the inability of SONs to generate action potentials during SWDs.

In all intracellularly recorded SONs, the start of the SWD in the EEG was correlated with an abrupt membrane hyperpolarization of mean amplitude $8.2 \pm 0.8 \mathrm{mV}$ (range, $6-12 \mathrm{mV} ; n=80$ SWDs from 10 cells) (Figs. $4 A, B 1,5 A, 6 A$ ), which persisted throughout the cortical seizure. As illustrated in Figure $4 A$ (vertical arrow), this negative shift in membrane potential could be reduced during a transient attenuation of the ictal EEG waves, demonstrating its functional link with the paroxysmal cortical oscillations. Rhythmic membrane depolarizations were superimposed on the tonic hyperpolarization (Figs. $4 A, B 1,5 A, 6 A$ ) and, despite their large amplitude $(22.8 \pm 0.2 \mathrm{mV}$; range, $9-39 \mathrm{mV}$; $n=888$ from 80 SWDs in 10 cells) (Fig. $4 A, B 1, C 1, C 2$ ), did not reach the voltage firing threshold, as measured by the intracellular injection of a threshold current pulse during interictal periods (Fig. 4B1,B2). Two major arguments suggest that these rhythmic membrane depolarizations originate synaptically (see also Discussion): (1) they were progressively sculpted by the temporal summation of high-frequency depolarizing potentials (Fig. 4C1), and (2) their frequency corresponded closely to that of spikewave complexes and was unchanged when membrane potential was modified by maintained current injection (data not shown).

The end of the SWD in the cortical EEG coincided with the cessation of intracellular striatal oscillations and removal of the tonic hyperpolarization (Fig. 4A). In six SONs, this postictal membrane repolarization was immediately followed by an abrupt depolarization leading to a membrane potential more positive than that preceding the ictal activity (Fig. $4 A$, oblique arrow). This postictal depolarization, measured from the preictal membrane potential to its peak depolarization, had an amplitude of $13.8 \pm 1.9 \mathrm{mV}$ (range, $10-23 \mathrm{mV} ; n=37$ SWDs from six cells) and decayed to preictal potentials within $1 \pm 0.2 \mathrm{sec}$. In two cells, one to three action potentials were generated corresponding to 
A

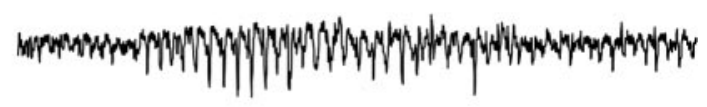
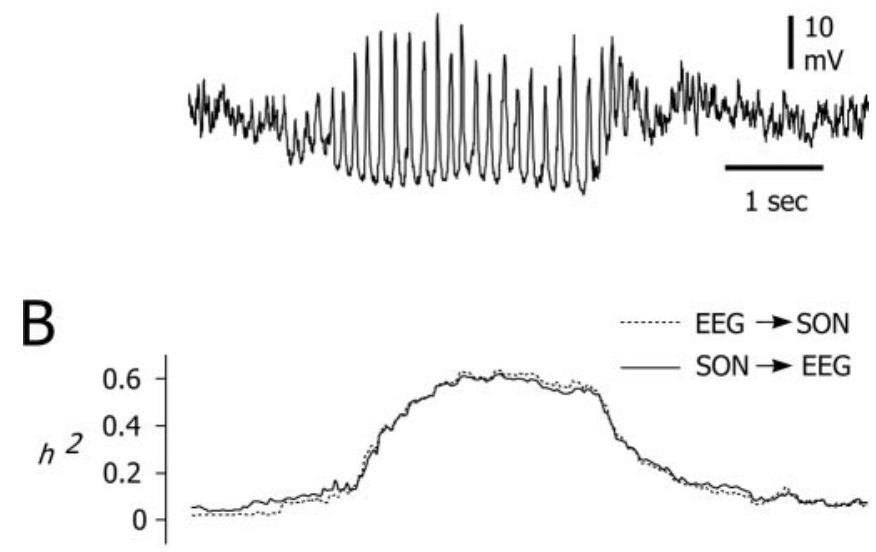

C

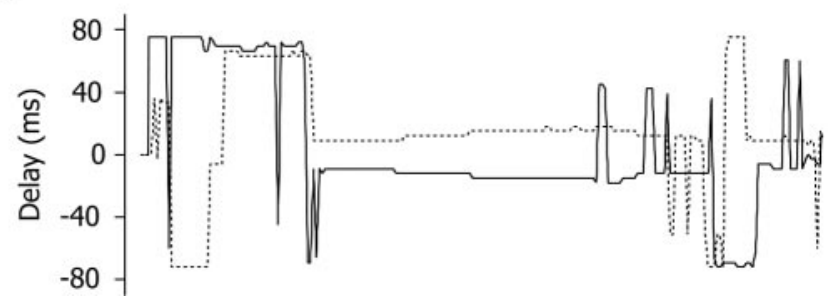

Figure 5. Temporal evolution of the relationships between EEG and intracellular activities of SONs. A, Typical EEG paroxysm (top trace) and its intracellular correlate in a simultaneously recorded SON (bottom trace). $B$, Time course of the strength of association between the cortical and striatal activities shown in $A$. The nonlinear correlation indices $h^{2}$ (EEG $\rightarrow$ SON, dashed line; SON $\rightarrow$ EEG, continuous line) were calculated for successive $2 \mathrm{sec}$ windows with a temporal overlapping of $98 \%$. C, Corresponding time delays. The time lag values fluctuated before and after the SWD but were relatively constant during the seizure. This result indicates a unidirectional coupling, from the cortex to the SONs, with a delay of $\sim 15 \mathrm{msec}$. The traces in $A$ and the corresponding analysis in $B$ and C are temporally aligned.

the postictal firing seen in extracellular recordings of SONs (Fig. 2A2).

\section{Association strength and temporal relationship between striatal intracellular oscillations and SWDs}

The strength of association ( $h^{2}$; see Material and Methods) between the EEG signal and intracellular activity in SONs increased as absence seizure developed. As illustrated in Figure $5 B$, the seizure onset was characterized by a gradual increase in the degree of association between the signals, and, after reaching a maximum that was maintained until the end of the crisis, $h^{2}$ progressively recovered its preictal value. In records from five SONs during 11 seizures, the average degree of association between intracellular and EEG signals during the first second of the SWD $\left(h^{2}=0.47 \pm\right.$ $0.08 ; n=11$ SWDs) was significantly higher than before the ictal event $\left(h^{2}=0.22 \pm 0.07 ; p<0.001\right)$. Furthermore, values for time delay extracted by nonlinear correlation of EEG waves, and the intracellular activity of SONs were profoundly modified by the absence seizure (Fig. 5C). Before SWDs, low values for $h^{2}$ indicated that the time delays were highly variable. In contrast, from the first second of the seizure, a clear time lag emerged between intracellular oscillations in SONs and cortical EEG waves and was maintained throughout the SWD (Fig. 5C). For 11 seizures analyzed, the mean delay was $11.4 \pm 4.7 \mathrm{msec}$.

\section{Chloride-dependent inhibition of SONs and activity of striatal GABAergic interneurons}

Why did SWD-associated rhythmic membrane depolarizations in SONs not generate action potentials? This could result from an insufficient excitatory synaptic drive to bring the cell to its firing threshold and/or from a shunting inhibition concomitant with the rhythmic excitation. One way to test for the presence of shunting inhibition is to search for changes in membrane input resistance associated with the activation of shunting synapses (Douglas et al., 1988; Pei et al., 1991). Thus, we applied repetitive (every $1.5 \mathrm{sec}$ ) positive square current pulses $(0.8-1 \mathrm{nA})$, and we examined, in two SONs, the voltage responses obtained from ictal and interictal periods (Fig. $6 A$ ). Because the $I-V$ relationship of the SONs membrane is not linear (Nisenbaum and Wilson, 1995) (Fig. 3), we compared responses induced from similar membrane potentials (Fig. 6B-D). According to this criterion, and given the tonic hyperpolarization of SONs during SWDs, the responses measured during SWDs were superimposed on the rhythmic membrane depolarizations (Fig. 6D). In the experiment illustrated in Figure $6 E$, the mean number of current-evoked action potentials was significantly $(p<0.001)$ reduced during the SWD-associated rhythmic depolarizations $(0.9 \pm 0.1$ spikes; $n=$ 10 stimulations $)$ compared with the interictal epochs $(2.35 \pm 0.9$ action potentials; $n=10$ stimulations). A similar result was obtained in another cell in which the number of current-induced action potentials was reduced by $76 \%$ during absence seizures (interictal, $1.78 \pm 0.15$ spikes vs ictal, $0.43 \pm 0.2$ spikes; $p<$ $0.0001)$. This decrease in current-evoked firing and the associated reduction of the underlying membrane depolarization (Fig. $6 E$, inset) show that membrane input resistance was decreased during the SWD-associated membrane depolarizations.

To test for a possible role of a chloride-dependent conductance in the decrease of the membrane resistance of SONs during rhythmic depolarizations, intracellular recordings were performed with $\mathrm{KCl}$-filled electrodes ( $n=5$ SONs). The interictal membrane potential of these neurons was more depolarized $(-63 \pm 0.12 \mathrm{mV} ; n=5$ cells; $p<0.001$; one-way ANOVA $)$ than that recorded with KAc-filled electrodes, although their apparent input resistance was lower $(23.7 \pm 0.7 \mathrm{M} \Omega ; n=3$ cells; $p<$ $0.001)$. Action potential amplitude $(57.3 \pm 3.8 \mathrm{mV} ; n=5$ cells $)$ and duration $(1.38 \pm 0.1 \mathrm{msec} ; n=5$ cells $)$ of $\mathrm{Cl}^{-}$-loaded SONs were similar to those recorded with KAc-filled electrodes $(p>$ 0.2 for each parameter). As observed with $\mathrm{KAc}$ electrodes, $\mathrm{Cl}^{-}$loaded SONs displayed during the cortical paroxysms a tonic membrane hyperpolarization $(5-15 \mathrm{mV})$ that lasted throughout the SWD (Fig. 7A). The postictal rebound of membrane depolarization was still observed and could trigger one (Fig. $7 A$, oblique arrow) or multiple action potentials. A remarkable finding was the conversion of the subthreshold oscillations into largeamplitude rhythmic depolarizations ( $n=3475$ from 84 SWDs) that could generate up to five action potentials ( $1.6 \pm 0.3$ spikes per depolarization; $n=3475$ depolarizations from five SONs) with a probability of firing of $0.6 \pm 0.3$ (Fig. $7 A, C$ ). The mean frequency and duration of the bursting activity, measured for at least two successive action potentials on a single depolarization, was $172 \pm 15 \mathrm{~Hz}$ (range, 131-201 Hz; $n=1181$ bursts from five SONs) and $9.8 \pm 1.2 \mathrm{msec}$ (range, $6.4-13.3 \mathrm{msec} ; n=1181$ bursts from five SONs), respectively. To assess the temporal relationship between the $\mathrm{Cl}^{-}$-dependent depolarization in SONs and the cortical paroxysms, we compared the timing of all action poten- 
tials, generated by the depolarizing $\mathrm{Cl}^{-}$dependent potentials, with that of the associated EEG spike (Fig. $7 B$, inset). The corresponding distribution of action potential delays was normally distributed around a mean value of $7.8 \pm 0.2 \mathrm{msec}$ ( $n=2357$ spikes from five SONs) (Fig. 7B).

When $\mathrm{Cl}^{-}$-loaded SONs were hyperpolarized by direct current (DC) injection $(n=2$ SONs $)$, at membrane potentials similar to or more negative than that of SONs recorded with KAc electrodes, the rhythmic depolarizations increased in amplitude and still induced action potential discharge (Fig. 7C). Thus, the rhythmic firing during SWDs did not result from the slight membrane depolarization observed in $\mathrm{Cl}^{-}$-loaded SONs (see above).

Our findings strongly suggest that the decrease in membrane resistance of SONs during SWDs resulted from an increase in $\mathrm{Cl}^{-}$conductance. Because SONs were normally silenced by the SWDs, this increase could not result from GABAergic $\mathrm{Cl}^{-}$-dependent interactions between SONs. It might instead originate from striatal GABAergic interneurones, which are known to produce a robust $\mathrm{Cl}^{-}$-dependent inhibition able to block the generation of action potentials in SONs (Plenz and Kitai, 1998; Koós and Tepper, 1999). We tested this hypothesis by performing extracellular recordings of striatal GABAergic interneurons $(n=5)$ during cortical SWDs. These cells were identified by their distinctive short-duration action potentials $(<1 \mathrm{msec})$ and their morphological characteristics, including a round soma (15-20 $\mu \mathrm{m}$ in diameter) and three to eight smooth primary dendrites (Kawaguchi, 1993) (Fig. 8A1). The irregular interictal firing of striatal GABAergic interneurons was converted during SWDs into highfrequency bursts $(2.15 \pm 0.3$ action potentials per burst; $n=5$ cells $)$ in-phase with the spike-wave complexes (Fig. 8A2,A3). The duration of bursts was $9.8 \pm 1.5 \mathrm{msec}$ (range, $4.6-12.5 \mathrm{msec} ; n=5$ cells), with an internal frequency of $224.4 \pm 23.1 \mathrm{~Hz}$ (range, 155-284 Hz; $n=5$ cells). The latency of the first action potential measured from the EEG spike component of the corresponding spike-wave complex was $1 \pm 0.3 \mathrm{msec}(n=1402$ spikes from five cells) (Fig. $8 \mathrm{~B}$ ). A similar analysis using all action potentials in a burst indicated a mean latency to the EEG spike of $4.1 \pm 0.2 \mathrm{msec}(n=3175$ spikes from 1402 bursts; $n=5$ cells) (Fig. $8 C$ ).

\section{Discussion}

The main findings of the present study, which provides the first description of the electrical events occurring in the corticostriatal pathway during absence seizures, are as follows: (1) CS neurons display suprathreshold rhythmic depolarizations in-phase with spike-wave complexes in the EEG, (2) the intracellular activity of SONs during SWDs is characterized by subthreshold membrane oscillations superimposed on a tonic hyperpolarization, and (3) SONs do not fire during absence seizures probably because of an increase in membrane $\mathrm{Cl}^{-}$conductance, which is temporally correlated with bursts of action potentials in striatal GABAergic interneurons. These results suggest that the propagation of cortical paroxysms through the corticostriatal pathway during ab- sence seizures might transiently decrease the synaptic inhibition of basal ganglia output nuclei.

\section{Sequential electrical events in the corticostriatal pathway during SWDs}

The occurrence of SWDs in the EEG was concomitant in CS neurons with an abrupt modification of activity similar to that previously described from a population of GAERS layer V cortical neurons with unidentified subcortical targets (Charpier et al., 1999). This finding indicates that the GAERS CS neurons were not a functionally distinct subset of cells but rather suggests that cortical output neurons exhibit a homogenous electrical behavior during SWDs. The small-amplitude irregular synaptic activity observed in CS neurons during the interictal period was converted during the SWD into rhythmic suprathreshold depolarizations. This rhythmic activity had the same frequency as that of the spike-wave complexes and was concomitant with a tonic membrane hyperpolarization that lasted for the whole duration of the SWD. Because this hyperpolarizing envelope was associated with an increase in the membrane input resistance (Charpier et al., 1999; Slaght et al., 2002b), it was probably attributable to a synaptic disfacilitation, i.e., a reduction of the tonic excitatory synaptic drive, rather than an active synaptic inhibition that should instead increase the membrane conductance (Paré et al., 1998; Timofeev et al., 2001). The frequency of membrane oscillations in CS neurons during SWDs was not modified by DCinduced changes in membrane potential, and their amplitude increased with membrane hyperpolarization (Charpier et al., 1999; Slaght et al., 2002b), strongly suggesting that they are generated by rhythmic depolarizing synaptic inputs. Because 
A
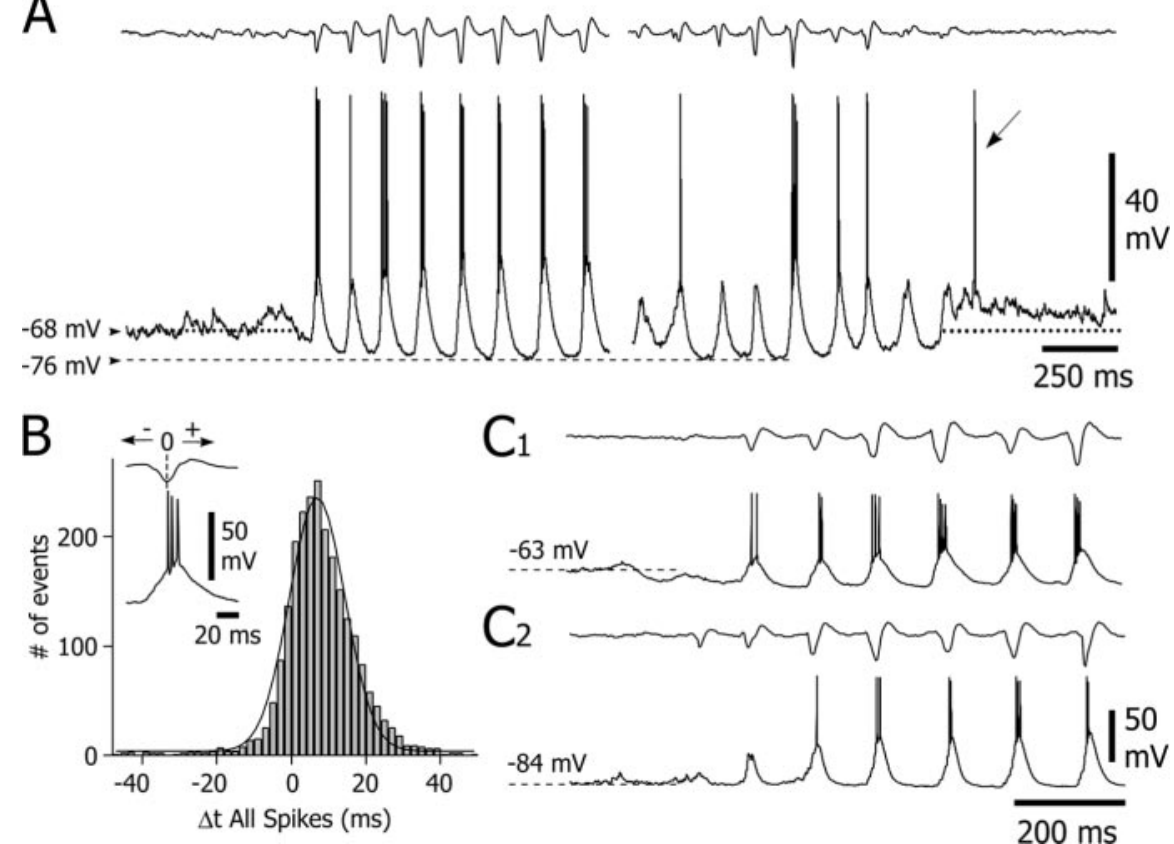

$\mathrm{C}_{2}$

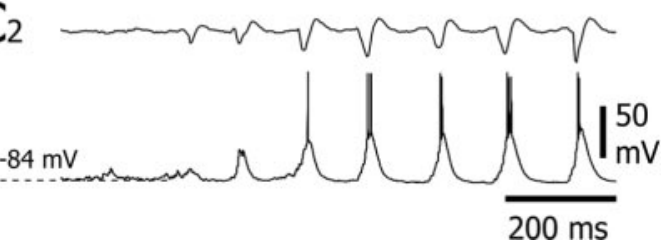

Figure 7. Intracellular activity of SONs recorded with KCl-filled microelectrodes. A, The start and the end of an SWD recorded from a $\mathrm{Cl}^{-}$-loaded SON. In contrast with the recordings obtained with KAc electrodes (see Figs. 4-6), SONs recorded with $\mathrm{KCl}$ electrodes (bottom trace) had an interictal membrane potential (dotted line) slightly more depolarized and, during SWDs (top trace), exhibited rhythmic depolarizing potentials that could generate bursts of action potentials. Two features were preserved: the tonic membrane hyperpolarization (dashed line) and the postictal rebound (oblique arrow). $B$, Distribution of the delays of the first action potential relative to the EEG spike component (inset). The pooled distribution $(n=2357$ action potentials from 5 cells; bin size, $2 \mathrm{msec}$; mean $=7.8 \pm 0.2 \mathrm{msec}$ ) was fitted by a Gaussian curve $\left(r^{2}=0.98\right) .\left(\right.$, Intracellular activity of a $\mathrm{Cl}^{-}$-loaded SON, at the start of an SWD, recorded from the resting potential (C1) and during maintained hyperpolarization to $-84 \mathrm{mV}$ by DC injection (C2). The frequency of the rhythmic depolarizations did not depend on the membrane polarization, and the cell still fired action potentials despite the substantial hyperpolarization. Records in $A$ and $C$ are from two different neurons.
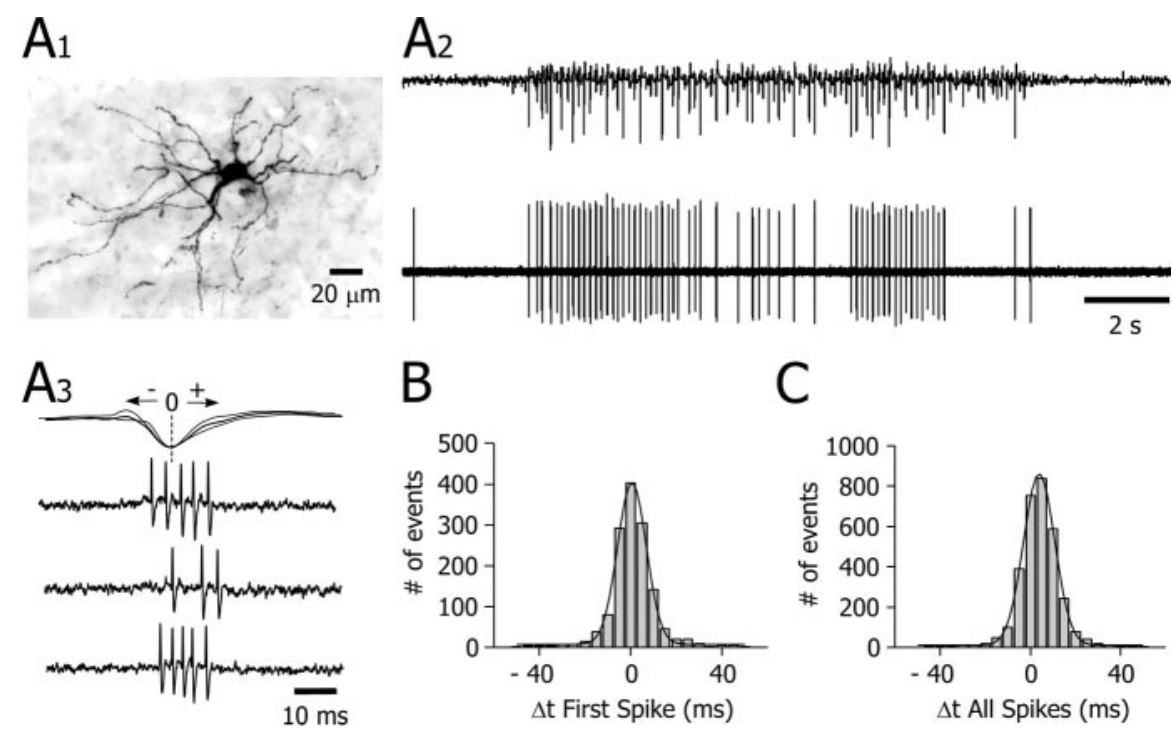

Figure 8. Bursting activity in GABAergic striatal interneurons during SWDs. A1, Synthetic projection micrograph (from a $400-\mu \mathrm{m}$-thick whole mount) of a GABAergic striatal interneuron labeled by juxtacellular injection of neurobiotin. This cell exhibited the distinctive morphological features (for a detailed description, see Results) of striatal interneurons immunoreactive for GABA (Kawaguchi, 1993). A2, A3, Extracellular recording of the labeled neuron shown in $A$. This cell displayed, during the cortical seizure ( $A 2$, top trace), recurrent bursts of action potentials ( $A 2$, bottom trace) concomitant with the spike-wave complexes in the EEG (A3). B, C, Histograms and Gaussian-Laplace fits (black lines) showing the latency $(\Delta t)$ of the first and all of the action potentials, respectively, in a burst with respect to the peak negativity of the EEG spike (taken as zero-time reference; $A 3$ ) ( $n=1402$ bursts from 5 cells; bin size, $5 \mathrm{msec}$ ). Note that the start of bursts was in-phase with the EEG spike $(B)$ and that most action potentials occurred $0-15$ msec after the EEG spike ( $($ ). large-amplitude EEG waves reflect a synchronization of synaptic potentials in many cortical neurons, and assuming a relatively homogeneous behavior of CS neurons during SWDs, our findings indicate that CS neurons are engaged, during absence seizures, in a tightly synchronized rhythmic excitation leading to coherent firing.

Both extracellular and intracellular recordings revealed that action potential discharge in SONs, which occurred irregularly and with a low frequency during interictal periods, was transiently interrupted during SWDs. This lack of firing was associated with subthreshold rhythmic depolarizations superimposed on a tonic hyperpolarization that lasted for the entirety of the cortical paroxysm. It is very unlikely that the sustained hyperpolarization resulted from a GABAergic synaptic inhibition because it was observed at membrane potentials more negative than the equilibrium potential of $\mathrm{Cl}^{-}$as measured in SONs $(\sim-60 \mathrm{mV})$ (Plenz, 2003) and was not reversed in polarity in chloride-loaded neurons. Alternatively, it could originate from a cessation of excitatory synaptic activity in SONs resulting from a transition in firing of their cortical afferents. The tonic firing of CS neurons during interictal activity could produce a sustained depolarization in SONs attributable to the summation of spontaneous excitatory synaptic events. The phasic synchronized firing in CS neurons during an SWD might allow, between spike-andwave complexes, a return to the SON resting potential, which depends strongly on inwardly rectifying potassium channels in the absence of strong depolarizing inputs (Nisenbaum and Wilson, 1995; Wilson and Kawaguchi, 1996).

Several lines of evidence strongly suggest that the synaptic oscillations in SONs during SWDs mainly result from rhythmic synchronized discharges in their cortical afferents. First, CS neurons provide powerful excitatory synaptic inputs to SONs (Kincaid et al., 1998; Zheng and Wilson, 2002) responsible for the large spontaneous synaptic depolarizations observed in these neurons in vivo (Wilson, 1995; Wilson and Kawaguchi, 1996; Stern et al., 1997; Charpier et al., 1999; Mahon et al., 2001). Second, the strength of association between cortical field potentials and synaptic depolarizations in SONs was dramatically increased during SWDs. Third, intracellular oscillations in SONs were delayed by $\sim 30$ msec with respect to the discharge of CS neurons (Fig. 9), a temporal shift consistent with the time required for integration of cortical synaptic potentials in SONs (Mahon et al., 2001). Al- 


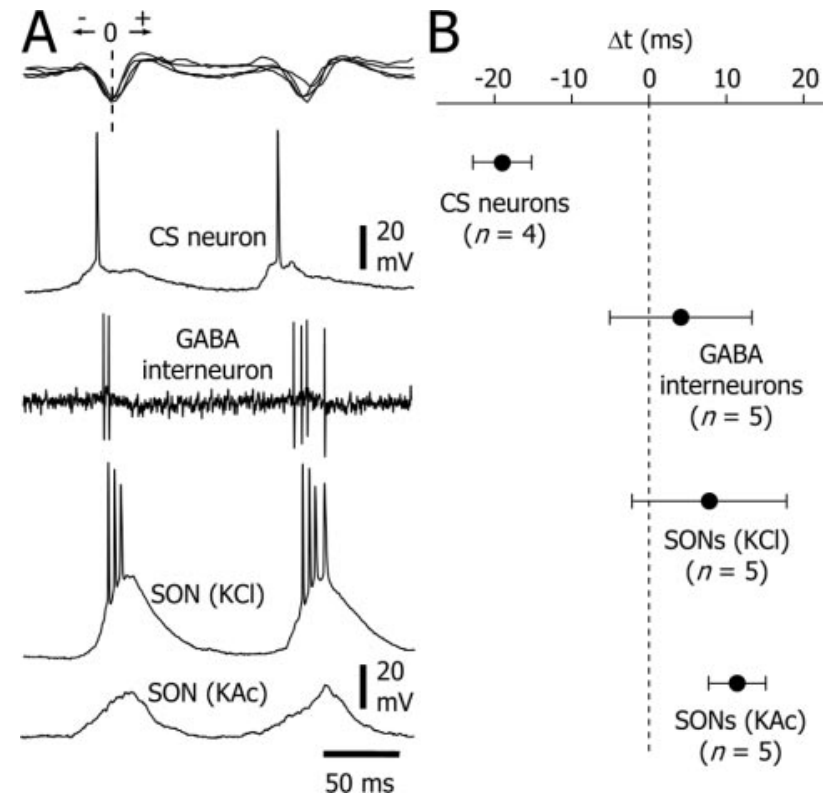

Figure 9. Temporal relationship between SWDs and associated cellular activities in the corticostriatal pathway. A, Examples of two successive EEG spike-wave complexes (top trace) and the corresponding intracellular activity in a CS neuron, an SON recorded with a KAc-filled electrode, an SON recorded with a $\mathrm{KCl}$-filled electrode, and the extracellular activity of a striatal GABAergic interneuron (bottom traces). The interneuron and the SON recorded with the KAcfilled electrode were recorded in the same animal. $B$, Averaged latencies (mean $\pm S D$ ) of all action potentials related to the EEG spike component used as the zero-time reference $(A$, dashed line). Because SONs recorded with KAc-filled electrodes were silent during SWDs, we used the time delays measured from the EEG and the intracellular waveforms (Fig. 5C). The number of computed neuron-EEG pairs is indicated in the figure.

though the activity of the excitatory thalamostriatal neurons (Wilson et al., 1983) during SWDs remains unknown, we cannot exclude their participation in the generation of synaptic oscillations in SONs.

An unexpected finding was that the rhythmic striatal depolarizations did not reach the firing threshold. These membrane oscillations were associated with an increase in membrane conductance and, in records with $\mathrm{KCl}$-filled electrodes, were increased in amplitude and could evoke bursts of action potentials. This $\mathrm{Cl}^{-}$dependent conductance in SONs, concomitant with the rhythmic excitatory inputs, may well result from the activity of striatal GABAergic interneurons that provide strong "feedforward" inhibition to SONs (Plenz and Kitai, 1998; Koós and Tepper, 1999). As illustrated in Figure 9, during SWDs, these interneurons discharged bursts of action potentials that followed the spiking of CS neurons at latencies of $\sim 20 \mathrm{msec}$ and coincided with the firing of $\mathrm{Cl}^{-}$-loaded SONs. Thus, it is likely that the powerful excitatory synaptic drive provided by the synchronized cortical inputs during SWDs generated bursting patterns in striatal interneurons. However, because thalamic inputs to these cells have been described (Sidibé and Smith, 1999), excitatory thalamostriatal connections may also be involved in the rhythmic excitation of striatal GABAergic interneurons during ictal activity.

Because the membrane potential reached in SONs during SWDs is markedly more negative than the equilibrium potential of $\mathrm{Cl}^{-}$(see above), GABA-mediated currents could, in principle, have a depolarizing action and therefore facilitate the rhythmic excitatory inputs originating from the cerebral cortex. However, it has been shown recently in pyramidal cortical neurons that such positive interactions between excitatory synaptic potentials and GABA-mediated depolarizing potentials is limited to time periods when the inhibitory conductance change has terminated but the associated membrane depolarization has not yet returned to rest (Gulledge and Stuart, 2003). In contrast, depolarizing inhibitory synaptic potentials have a powerful inhibitory action when excitatory inputs occur simultaneously with the GABA-mediated conductance change (Staley and Mody, 1992; Gulledge and Stuart, 2003). This was the case in the present study in which we found a close temporal overlap during SWDs, between firing in GABAergic interneurons and the rising phase of the rhythmic depolarizations in SONs (Fig. 9). Therefore, although GABA responses in SONs can be depolarizing during SWDs, the increase in membrane conductance, coincident with the cortical inputs, might produce a shunting inhibition that limits membrane depolarization positive to the equilibrium potential of $\mathrm{Cl}^{-}$and, consequently, prevents SON firing.

The present experiments do not provide a straightforward explanation for the rebound of excitation observed in SONs at the end of the SWDs. However, it could result from a postictal increase in excitability in thalamic and/or cortical neurons (Grenier et al., 1998; Timofeev et al., 2002) afferent to the striatum.

\section{Possible implications for the control of absence seizures}

The main finding of the present study is the transient interruption of firing in SONs during absence seizures, which presumably decreases the synaptic inhibition of their targets in the SNr. This should modify the balance between synaptic inhibition and excitation of the $\mathrm{SNr}$ neurons, tending to reinforce the actions of excitatory synaptic inputs arising from the subthalamic nucleus (Kita, 1994). This hypothesis is supported by a recent study in freely moving GAERS showing a synchronized bursting firing of $\mathrm{SNr}$ neurons in-phase with the spike-wave complexes in the EEG (Deransart et al., 2003).

The possibility that absence seizures are modulated by the GABAergic striatonigral pathway has emerged from pharmacological studies in GAERS, suggesting that an increase or a decrease in the activity of the striatum could reduce or amplify, respectively, the occurrence of SWDs (Depaulis et al., 1988, 1989; Danober et al., 1998; Deransart et al., 1998, 2000, 2001; Deransart and Depaulis, 2002) (see Introduction). Changes in the output of SONs associated with the occurrence and the termination of the SWD could be part of endogenous mechanisms controlling the maintenance and the duration of the abnormal oscillations in the thalamocortical loops during absence seizures. First, the lack of spike discharge in SONs during SWDs should facilitate the bursting activity of SNr neurons and so tend to prolong the seizure. Second, the rebound of excitation that we observed in SONs at the end of SWDs, which is consistent with the decrease of SNr activity at the end of cortical paroxysms (Deransart et al., 2003), could contribute to seizure termination.

\section{References}

Bruno RM, Khatri V, Land PW, Simons DJ (2003) Thalamocortical angular tuning domains within individual barrels of rat somatosensory cortex. J Neurosci 23:9565-9574.

Chang HT, Wilson CJ, Kitai ST (1982) A Golgi study of rat neostriatal neurons: light microscopic analysis. J Comp Neurol 208:107-126.

Charpier S, Lereshe N, Deniau J-M, Mahon S, Hughes SW, Crunelli V (1999) On the putative contribution of $\mathrm{GABA}_{\mathrm{B}}$ receptors to the electrical events occurring during spontaneous spike and waves discharges. Neuropharmacology 38:1699-1706.

Connors BW, Gutnick MJ (1990) Intrinsic firing patterns of diverse neocortical neurons. Trends Neurosci 13:99-104.

Cowan RL, Wilson CJ (1994) Spontaneous firing patterns and axonal projections of single corticostriatal neurons in the rat medial agranular cortex. J Neurophysiol 71:17-32.

Crunelli V, Leresche N (2002) Childhood absence epilepsy: genes, channels, neurons and networks. Nat Rev Neurosci 3:371-382.

Czubayko U, Plenz D (2002) Fast synaptic transmission between striatal spiny projection neurons. Proc Natl Acad Sci USA 99:15764-15769. 
Danober L, Deransart C, Depaulis A, Vergnes M, Marescaux C (1998) Pathophysiological mechanisms of genetic absence epilepsy in the rat. Prog Neurobiol 55:27-57.

Deniau JM, Menetrey A, Charpier S (1996) The lamellar organization of the rat substantia nigra pars reticulata: segregated patterns of striatal afferents and relationship to the topography of corticostriatal projections. Neuroscience 73:761-781.

Depaulis A, Vergnes M, Marescaux C, Lannes B, Warter J (1988) Evidence that activation of GABA receptors in the substantia nigra suppresses spontaneous spike-and-wave discharges in the rat. Brain Res 448:20-29.

Depaulis A, Snead OI, Marescaux C, Vergnes M (1989) Suppressive effects of intranigral injection of muscimol in three models of generalized nonconvulsive epilepsy induced by chemical agents. Brain Res 498:64-72.

Deransart C, Depaulis A (2002) The control of seizures by the basal ganglia? A review of experimental data. Epileptic Disord 4 [Suppl 3]:S61-S72.

Deransart C, Vercueil L, Marescaux C, Depaulis A (1998) The role of basal ganglia in the control of generalized absence seizures. Epilepsy Res 32: 213-223.

Deransart C, Riban V, Le B, Marescaux C, Depaulis A (2000) Dopamine in the striatum modulates seizures in a genetic model of absence epilepsy in the rat. Neuroscience 100:335-344.

Deransart C, Le-Pham BT, Hirsch E, Marescaux C, Depaulis A (2001) Inhibition of the substantia nigra suppresses absences and clonic seizures in audiogenic rats, but not tonic seizures: evidence for seizure specificity of the nigral control. Neuroscience 105:203-211.

Deransart C, Hellwig B, Heupel-Reuter M, Leger JF, Heck D, Lucking (2003) Single-unit analysis of substantia nigra pars reticulata neurons in freely behaving rats with genetic absence epilepsy. Epilepsia 44:1513-1520.

Douglas RJ, Martin KA, Whitteridge D (1988) Selective responses of visual cortical cells do not depend on shunting inhibition. Nature 332:642-644.

Grenier F, Timofeev I, Steriade M (1998) Leading role of thalamic over cortical neurons during postinhibitory rebound excitation. Proc Natl Acad Sci USA 95:13929-13934.

Gulledge AT, Stuart GJ (2003) Excitatory actions of GABA in the cortex. Neuron 37:299-309.

Kawaguchi Y (1993) Physiological, morphological, and histochemical characterization of three classes of interneurons in rat neostriatum. J Neurosci 13:4908-4923.

Kincaid AE, Zheng T, Wilson CJ (1998) Connectivity and convergence of single corticostriatal axons. J Neurosci 18:4722-4731.

Kita H (1993) GABAergic circuits of the striatum. Prog Brain Res 99:51-72.

Kita H (1994) Physiology of two disynaptic pathways from the sensorimotor cortex to the basal ganglia output nuclei. In: The basal ganglia IV (Percheron G, McKenzie JS, Féger J, eds), pp 263-276. New York: Plenum.

Koós T, Tepper JM (1999) Inhibitory control of neostriatal projection neurons by GABAergic interneurons. Nat Neurosci 2:467-472.

Lopes da Silva F, Pijn JP, Boeijinga P (1989) Interdependence of EEG signals: linear vs. nonlinear associations and the significance of time delays and phase shifts. Brain Topogr 2:9-18.

Mahon S, Deniau JM, Charpier S (2001) Relationship between EEG potentials and intracellular activity of striatal and cortico-striatal neurons: an in vivo study under different anesthetics. Cereb Cortex 11:360-373.

Mahon S, Casassus G, Mulle C, Charpier S (2003) Spike-dependent intrinsic plasticity increases firing probability in rat striatal neurons in vivo. J Physiol (Lond) 550:947-959.

Mailly P, Charpier S, Menetrey A, Deniau JM (2003) Three-dimensional organization of the recurrent axon collateral network of the substantia nigra pars reticulata neurons in the rat. J Neurosci 23:5247-5257.

Marescaux C, Vergnes M, Depaulis A (1992) Genetic absence epilepsy in rats from Strasbourg - a review. J Neural Transm Suppl 35:37-69.

Meeren HK, Pijn JP, Van Luijtelaar EL, Coenen AM, Lopes da Silva FH (2002) Cortical focus drives widespread corticothalamic networks during spontaneous absence seizures in rats. J Neurosci 22:1480-1495.

Neafsey EJ, Bold EL, Hass G, Hurley-Gius KM, Quirk G, Sievert CF, Terreberry RR (1986) The organization of the rat motor cortex: a microstimulation mapping study. Brain Res Rev 11:77-96.

Nisenbaum ES, Wilson CJ (1995) Potassium current responsible for inward and outward rectification in rat neostriatal spiny projection neurons. J Neurosci 15:4449-4463.

Panayiotopoulos CP (1997) Absences epilepsies. In: Epilepsy: a comprehen- sive textbook (Engel Jr J, Pedley TA, eds), pp 2327-2346. Philadelphia: Lippincott-Raven.

Paré D, Shink E, Gaudreau H, Destexhe A, Lang EJ (1998) Impact of spontaneous synaptic activity on the resting properties of cat neocortical pyramidal neurons in vivo. J Neurophysiol 79:1450-1460.

Pei X, Volgushev M, Vidyasagar TR, Creutzfeldt OD (1991) Whole cell recording and conductance measurements in cat visual cortex in-vivo. NeuroReport 2:485-488.

Pinault D (1996) A novel single-cell staining procedure performed in vivo under electrophysiological control: morpho-functional features of juxtacellularly labeled thalamic cells and other central neurons with biocytin or Neurobiotin. J Neurosci Methods 65:113-136.

Pinault D (2003) Cellular interactions in the rat somatosensory thalamocortical system during normal and epileptic 5-9 Hz oscillations. J Physiol (Lond) 552:881-905.

Pinault D, Lereshe N, Charpier S, Deniau JM, Marescaux C, Vergnes M, Crunelli V (1998) Intracellular recordings in thalamic neurones during spike and waves discharges in rats with absence epilepsy. J Physiol (Lond) 509:449-456.

Plenz D (2003) When inhibition goes incognito: feedback interaction between spiny projection neurons in striatal function. Trends Neurosci 26:436-443.

Plenz D, Kitai ST (1998) Up and down states in striatal medium spiny neurons simultaneously recorded with spontaneous activity in fast-spiking interneurons studied in cortex-striatum-substantia nigra organotypic cultures. J Neurosci 18:266-283.

Rall W (1969) Time constants and electrotonic length of membrane cylinders and neurons. Biophys J 9:1483-1508.

Ramanathan S, Hanley JJ, Deniau JM, Bolam JP (2002) Synaptic convergence of motor and somatosensory cortical afferents onto GABAergic interneurons in the rat striatum. J Neurosci 22:8158-8169.

Sidibé M, Smith Y (1999) Thalamic inputs to striatal interneurons in monkeys: synaptic organization and co-localization of calcium binding proteins. Neuroscience 89:1189-1208.

Simons DJ, Carvell GE (1989) Thalamocortical response transformation in the rat vibrissa/barrel system. J Neurophysiol 61:311-330.

Slaght SJ, Leresche N, Deniau JM, Crunelli V, Charpier S (2002a) Activity of thalamic reticular neurons during spontaneous genetically determined spike and wave discharges. J Neurosci 22:2323-2334.

Slaght SJ, Paz T, Mahon S, Maurice N, Charpier S, Deniau JM (2002b) Functional organization of the circuits connecting the cerebral cortex and the basal ganglia: implications for the role of the basal ganglia in epilepsy. Epileptic Disord 4 [Suppl 3]:S9-S22.

Staley KJ, Mody I (1992) Shunting of excitatory input to dentate gyrus granule cells by a depolarizing GABAA receptor-mediated postsynaptic conductance. J Neurophysiol 68:197-212.

Stern EA, Kincaid AE, Wilson CJ (1997) Spontaneous subthreshold membrane potential fluctuations and action potential variability of rat corticostriatal and striatal neurons in vivo. J Neurophysiol 77:1697-1715.

Timofeev I, Grenier F, Steriade M (2001) Disfacilitation and active inhibition in the neocortex during the natural sleep-wake cycle: an intracellular study. Proc Natl Acad Sci USA 98:1924-1929.

Timofeev I, Bazhenov M, Sejnowski T, Steriade M (2002) Cortical hyperpolarization-activated depolarizing current takes part in the generation of focal paroxysmal activities. Proc Natl Acad Sci USA 98:9533-9537.

Tunstall MJ, Oorschot DE, Kean A, Wickens JR (2002) Inhibitory interactions between spiny projection neurons in the rat striatum. J Neurophysiol 88:1263-1269.

Williams DA (1953) A study of thalamic and cortical rhythms in petit mal. Brain 76:56-69.

Wilson CJ (1995) The contribution of cortical neurons to the firing pattern of striatal spiny neurons. In: Models of information processing in the basal ganglia (Houk JC, Davies JL, Beiser DG, eds), pp 29-50. Cambridge: MIT.

Wilson CJ, Kawaguchi Y (1996) The origins of two-state spontaneous membrane potential fluctuations of neostriatal spiny neurons. J Neurosci 16:2397-2410.

Wilson CJ, Chang HT, Kitai ST (1983) Origins of post synaptic potentials evoked in spiny neostriatal projection neurons by thalamic stimulation in the rat. Exp Brain Res 51:217-226.

Zheng T, Wilson CJ (2002) Corticostriatal combinatorics: the implications of corticostriatal axonal arborizations. J Neurophysiol 87:1007-1017. 